\title{
Temporal Gradient based Satellite Image Compression
}

\author{
Sanchita Rani Das \\ Department of Computer Science \\ \& Engineering, \\ Prime University, \\ Bangladesh
}

\author{
Md. Al Mamun \\ Department of Computer Science \\ \& Engineering, \\ Rajshahi University of Engineering \\ \& Technology, \\ Bangladesh
}

\author{
Md. Ali Hossain \\ Department of Computer Science \\ \& Engineering, \\ Rajshahi University of Engineering \\ \& Technology, \\ Bangladesh
}

\begin{abstract}
Now a day's tremendous amount of earth observing data or images is downloaded in order to accommodate in variety of geographical and environmental applications like change detection, weather forecasting, climate change, disaster management etc. The recent advancement in spatial, spectral and temporal resolution of satellite images has make it possible to use the images in these vital and world-wide challenging applications. Due to limited transmission rate, remote sensed satellite images needs to be compressed before being delivered to the user. While individual images can be processed by considering spatial and spectral redundancies, a communication system for satellite images can also consider the temporal correlation between images of same place for two different time. In this paper a gradient-based temporal compression technique has been proposed to approximate the image using a reference image of previous time, which is already available. The residual image is transmitted where the proposed rate defeats the sate of art compression technology like JPEG.
\end{abstract}

\section{General Terms}

Compression, Entropy, Satellite image, Prediction

\section{Keywords}

Correlation, Gradient, Landsat ETM+, Multi-temporal

\section{INTRODUCTION}

Remote sensed satellite images gathers plenty of information which can be used in variety of geographical and environmental applications like change detection, weather forecasting, climate change, disaster management etc. The recent advancement in spatial, spectral and temporal resolution of satellite images has make it possible to use the images in these vital and world-wide thought-provoking applications. Petabytes of data/images are now available to be downloaded and thus satellite image communication or compression has become a critical issue in remote sensed data management. Lossy compression is preferable where a certain amount of image alteration is tolerable. It is not suitable for continuous data communication system as the recent data/image needs to be fully reconstructed and can be used as a reference as well [1]. Moreover, some application can't afford loss because every pixel has sensitive information. Lossless compression of satellite images have been classified into some major area of research methodologies, including prediction based, transformation based, and clustering based. The performance of the prediction-based approach depends on designing accurate predictor where residual image entropy is lower than the original one and the compression ratio is higher. Median predictor is being used in [2] whereas residual prediction is performed at the last stage between two stages. Apart from spatial or spectral de-correlations [3][4], temporal de-correlation has also been introduced [1][5]. The reference image in temporal prediction is assumed to be linearly related to the other images in the time sequence if there is no significant land cover change.

A lossless compression approach has been proposed in this paper that uses gradient-based temporal prediction to approximate the image using a reference image of previous time, which is already available. Most of the time anomalous pixels are considered as outlier for spatial or spectral prediction based compression approach and they are dealt separately [6][7]. Similarly the unpredictable pixels of multitemporal images need to be removed and the pixels having different temporal features compared with the same location pixels in the reference image are reformed [8]. That is why gradient-based compression approaches show high adaptive ability compared with other approaches as it can easily classify the unpredictable pixels as temporal edges. The median edge detector in MED prediction technique categorizes the horizontal and vertical edges. In spatial prediction considering local neighbourhood it predicts the current pixel from pixels that are not part of the edges [9](Figure. 1). This idea is used in spectral prediction [3][4][10] and extended for multi-temporal image compression in this paper.

\section{PREDICTION METHOD}

If any edge is detected in the reference image, it is highly possible that it will also occur in the recent image. Consider the neighbourhood from the previous image and the recent image in Figure. 2(a) and Figure. 2(b), the gradient-based temporal predictor is proposed as follows:

$$
\begin{aligned}
& \text { 1. if }|x(i, j) \quad x(i \quad 1, j)||x(i, j) \quad x(i, j \quad 1)|>\text { then } \\
& \text { predicted } y(i, j)^{*}=y(i, j \quad 1)+\{x(i, j) \quad x(i, j \quad 1)\} \\
& \text { 2. else if }|x(i, j) \quad x(i \quad 1, j)||x(i, j) \quad x(i, j \quad 1)|<\quad \text { then } \\
& \text { predicted } y(i, j)^{*}=y(i \quad 1, j)+\{x(i, j) \quad x(i \quad 1, j)\}
\end{aligned}
$$

where $\delta$ is a threshold and $\alpha$ is the linear regression parameter when executed between reference image and recent image. The first equation is meant to identify the horizontal edge and the second one to identify the sharp vertical edge in the reference image. 

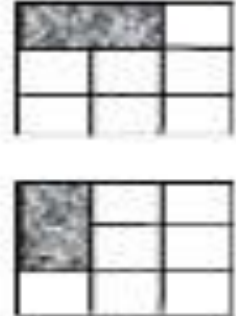

Fig 1: Edges in spatial prediction (horizontal and vertical).

\begin{tabular}{|l|l|l|}
\hline$x(i-1, j-1)$ & $x(i-1, j)$ & $x(i-1, j+1)$ \\
\hline$x(i, j-1)$ & $x(i, j)$ & \\
\hline & & \\
& & \\
\hline
\end{tabular}

(a)

\begin{tabular}{|l|l|l|}
\hline$y(i-1, j-1)$ & $y(i-1, j)$ & $y(i-1, j+1)$ \\
\hline$y(i, j-1)$ & $y(i, j)$ & \\
\hline & & \\
& & \\
\hline
\end{tabular}

(b)
Fig 2: Pixel localization (a) reference image (old), (b) recent image

\section{MULTI-TEMPORAL IMAGE COMPRESSION}

Consider two

image

set,

$Z=\left\{X=x_{i}, Y=y_{i} \mid i=1, \ldots, n\right\}$, where $X$ and $Y$ are captured at two different time $\mathrm{t}$ and $\mathrm{t}+1$, respectively, and $\mathrm{n}$ is the number of pixels, a prediction function, $\psi($.$) , and a$ residual/error function, $d($. $) \geq 0$, temporal prediction can be derived as the minimization problem.

$\psi^{\text {opt }}=\underset{\psi \in \lambda}{\operatorname{argmin}} \sum_{i=1}^{n} d\left(\psi\left(x_{i}\right), y_{i}\right)$

where $d\left(\psi\left(x_{i}\right), y_{i}\right)=\left(\psi\left(x_{i}\right)-y_{i}\right)^{2}$ is the sum squared errors or in short residuals between the predicted value and the exact value, and $\lambda$ a set of possible prediction functions. The minimization or optimization procedure (Equation. (1)) is to find the prediction model that will minimize the residual or errors between the predicted and the actual values of the dependent variable, Y.

Multi-temporal compression gain is very high due to the large temporal correlation that exists between the two images that are sometime apart. Actually temporal correlation coefficient indicates the strength of the relationship between variables or in this case it shows how two temporal images vary together. It actually quantifies the linear consistency between the images. The correlation coefficient between two dates' image data sets $X=\{x\}$ and $Y=\{y\}$, is defined as

$$
r_{X Y}=\frac{\sum_{i=1}^{n}\left(x_{i}-\bar{x}\right)\left(y_{i}-\bar{y}\right)}{\sqrt{\sum_{i=1}^{n}\left(x_{i}-\bar{x}\right)^{2} \sum_{i=1}^{n}\left(y_{i}-\bar{y}\right)^{2}}}
$$

where $\bar{x}$ and $\bar{y}$ are the sample means of $X$ and $Y$, respectively, and $\mathrm{n}$ the total number of pixels. However, the prediction function can be selected conferring the association shown by the temporal correlation and in the proposed system the high temporal correlation justifies the use of gradient based prediction approach describes in section II as the candidate function, $\psi($.$) .$

\section{IMAGES}

Resolution of the sensors installed in the satellite is developing in to a dominant level. Spatial resolution considered as the size corresponding to the ground area covered by a pixel. Landsat ETM+ (enhanced thematic mapper) captures images in medium spatial resolution, which is measured as 15-80 meters. The images are multispectral image with the spectral range of $0.45 \mu \mathrm{m}$ to $2.35 \mu \mathrm{m}$, which include one panchromatic band and one thermal band of different resolution. In the proposed experiment, 6 bands of Landsat ETM+ images of the central capital region of Australia were captured where the images are one year apart of which 3 bands are shown in Figure. 3, Figure. 4 and Figure. 5 for band1, band 2 and band 3 respectively. The 3D scatter plot for band 3 images between the two dates is shown in Fig. 6 . The high dense clutter clearly indicates the high association between the images. All the 6 bands have high temporal correlations between the two dates that can be easily shown in Figure 7. Band 6 and 8 are omitted because these have different resolution than the other bands. The final results by applying the proposed method have been discussed in the following section.

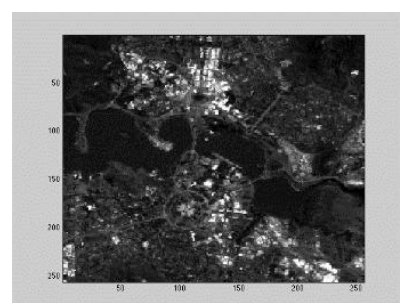

(a)

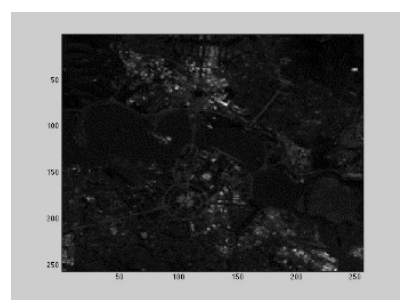

(b)
Fig 3: Satellite (Landsat Enhanced Thematic Map+) images (band1) captured in year (a) 2000 and (b) 2001.

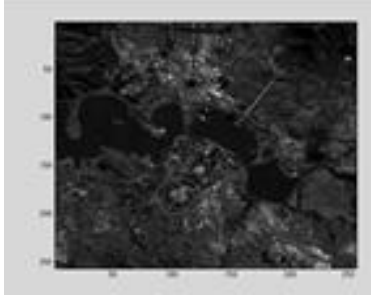

(a)

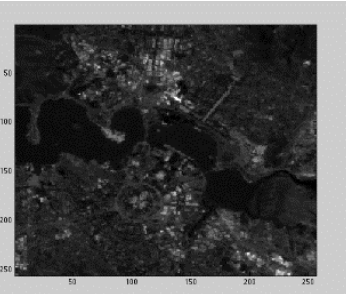

(b)
Fig 4: Satellite (Landsat Enhanced Thematic Map+) images (band2) captured in year (a) 2000 and (b) 2001.

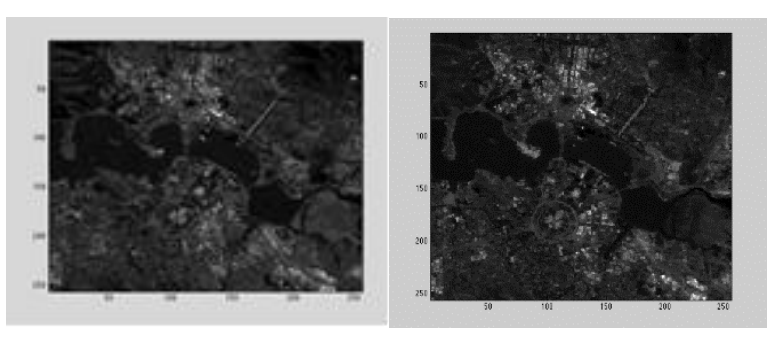

(a)

(b)

Fig 5: Satellite (Landsat Enhanced Thematic Map+) images (band3) captured in year (a) 2000 and (b) 2001. 


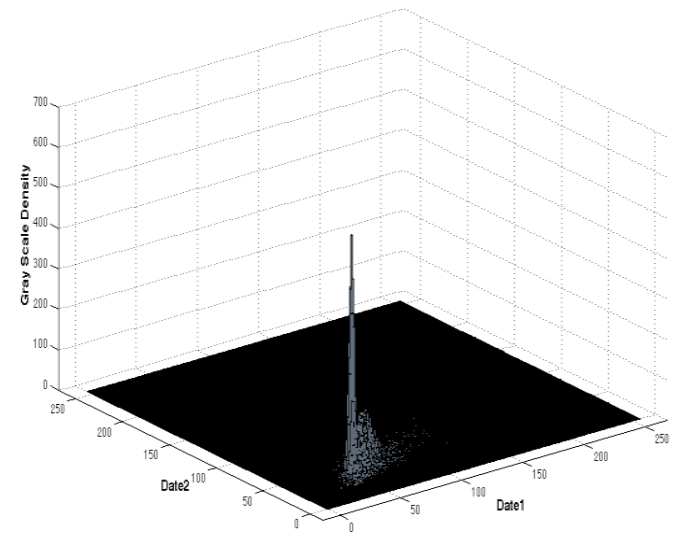

Fig 6: 3D scatter plot between the images for band3 captured in the year 2000 and 2001.

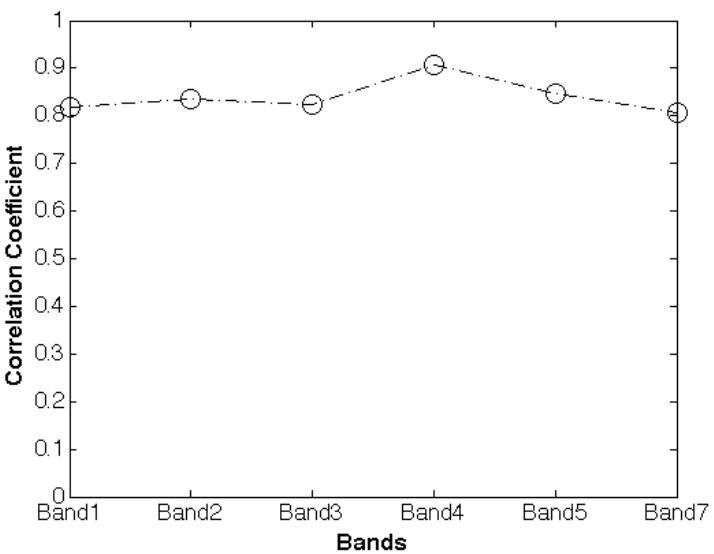

Fig 7: Temporal Correlation coefficients for all the bands.

\section{RESULTS AND DISCUSSATION}

The significant performance measurement in this case is known as entropy, which is the minimum number of bits required for the storage and transmission of the image. Let $x_{i}$ be a data set over a space, $X$, so $x_{i} \in\{X\}$ where $\mathrm{i}=1$ to $\mathrm{n}$. The entropy of $x_{i}$ is the function of its distribution. If $P($. is the probability mass function defined over $X$, the entropy of $X$ is

$$
H(X)=\sum_{i=1}^{n} p\left(x_{i}\right) \log _{2} \frac{1}{p\left(x_{i}\right)}
$$

The entropy is actually dependent upon the likelihood of the distribution of the pixel values. Whenever a good prediction model is realized, the overall prediction errors can be closely guessed by the symmetric narrow exponential distribution. The entropy of this kind of distribution is assumed to be monotonic function of its variance: The smaller the variance, the smaller the entropy [13].

Firstly the original entropy of the recent or current image has been calculated together with the entropy of the compressed image when JPEG 2000 is applied. These measures are then compared with the residual entropy of the proposed method. Here linear implementation [14] of the temporal compression has also been used for comparison.
Considering $\lambda$ in Equation. 1 as a general polynomial function defined as

$$
\lambda=\alpha_{0}+\alpha_{1} x^{1}+\alpha_{2} x^{2}+\ldots \ldots \ldots \ldots \ldots+\alpha_{p} x^{p}
$$

Here $\mathrm{p}$ is the degree of the polynomial, when $\mathrm{p}>1, \lambda$ is a non-linear function. For frequent temporal images, it is not unrealistic to assume that the non-changed pixels will have identical brightness values. Even if the images are some days apart. Considering all these facts first-order polynomial is used as the linear prediction function for images, which contain a small portion of real changes. Taking $\alpha_{0}=\beta$ and, $\alpha_{1}=\alpha$ for the first-order polynomial, the candidate prediction function becomes [14],

$$
\psi\left(x_{i}\right)=\alpha x_{i}+\beta
$$

The entropy comparison for all possible way of image distribution have been shown in Table I and corresponding graph has been shown in Figure. 8. The results easily depicts that the proposed method outperform the state of art technology JPEG 2000. The linear prediction performs well with respect to the original image transmission but can not beat JPEG 2000. This is due to the fact that the temporal edges can only be handled through the proposed robust gradient based approach which is ignored in linear approaches.

Table 1: Comparison with the state of art methods

\begin{tabular}{|c|c|c|c|c|}
\hline \multicolumn{5}{|c|}{ Entropy } \\
\hline Bands & $\begin{array}{c}\text { Original } \\
\text { Entropy }\end{array}$ & $\begin{array}{c}\text { JPEG } \\
\text { 2000 }\end{array}$ & $\begin{array}{c}\text { Linear } \\
\text { Prediction }\end{array}$ & $\begin{array}{c}\text { Proposed } \\
\text { Method }\end{array}$ \\
\hline B1 & 4.4745 & 3.5901 & 3.6874 & 3.4965 \\
\hline B2 & 4.7903 & 3.5803 & 3.8775 & 3.4730 \\
\hline B3 & 5.3158 & 4.0173 & 4.4462 & 3.9201 \\
\hline B4 & 5.8456 & 4.2670 & 4.8022 & 4.1327 \\
\hline B5 & 6.0473 & 4.7086 & 5.1049 & 4.5910 \\
\hline B7 & 5.7295 & 4.4889 & 4.7860 & 4.3705 \\
\hline
\end{tabular}

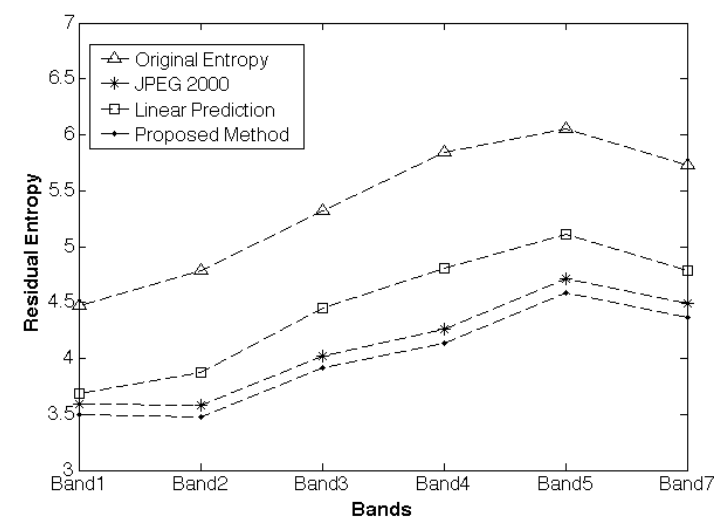

Fig 8: Entropy comparison for possible ways of transmission.

\section{CONCLUSION}

The temporal compression of optical multispectral image data has been introduced in this paper. The previous approaches mostly adopted compression approaches considering spatial and spectral correlations or redundancies. Whereas this paper explores a new area of investigation in individual data set compression by reflecting temporal correlations. In short the 
sequential data compression approach is motivated by the temporal correlation observed between two images in different time sequence. Although de-correlation in the spectral and spatial domains is the widely adopted technique for individual image-based compression, higher compression gains can be achieved by integrating them with de-correlation in the temporal domain.

\section{REFERENCES}

[1] Mamun M.A., Jia X. and Ryan M., "Non-linear Elastic Model for Flexible Prediction of Remote Sensed Multitemporal images". IEEE Geoscience and Remote Sensing Letters, vol. 11, no. 5, pp. 1005 - 1009, May 2014.

[2] Cheng-Chen, L., and Yin-Tsung, H. (2010). "An Efficient Lossless Compression Scheme for Hyperspectral Images Using Two-Stage Prediction." IEEE Geoscience and Remote Sensing Letters, 7(3), 558562.

[3] Magli E., Olmo G., and Quacchio E., "Optimized onboard lossless and near- lossless compression of hyperspectral data using CALIC." IEEE Geoscience and Remote Sensing Letters, 1(1), 21-25, 2004.

[4] Magli E., "Multiband Lossless Compression of Hyperspectral Images." IEEE Transactions on Geoscience and Remote Sensing, 47(4), 1168-1178, 2009.

[5] Wei Z., Qian D. and Fowler J.E., "Multitemporal hyperspectral image compression." IEEE Geoscience and Remote Sensing Letters, vol. 8(3), pp. 416-420, 2011.

[6] Qian D., Fowler J. E., and Wei Z., "On the Impact of Atmospheric Correction on Lossy Compression of Multispectral and Hyperspectral Imagery." IEEE Transactions on Geoscience and Remote Sensing, 47(1), 130-132, 2009.

[7] Penna B., Tillo T., Magli E., and Olmo G., "Hyperspectral Image Compression Employing a Model of Anomalous Pixels." IEEE Geoscience and Remote Sensing Letters, 4(4), 664-668, 2007.

[8] Mamun M. A., Jia X. and Hossain M. A. "Reconstruction of Satellite Images by Multi-temporal Gradient based Sequential Prediction", IEEE Geoscience and Remote Sensing Symposium (IGARSS '14), pp. 1616-1618, Quebec, Canada, July 2014.

[9] Weinberger M. J., Seroussi G., and Sapiro G., "The LOCO-I lossless image compression algorithm: principles and standardization into JPEG-LS." IEEE Transactions on Image Processing, 9(8), 1309-1324, 2000.

[10] Magli E., Olmo G., and Quacchio E., "Optimized onboard lossless and near- lossless compression of hyperspectral data using CALIC." IEEE Geoscience and Remote Sensing Letters, 1(1), 21-25, 2004

[11] Hongqiang W., Babacan S. D., and Sayood K., "Lossless hyperspectral image compression using context-based conditional averages." Data Compression Conference Proceedings (DCC '05), 418-426, 2005.

[12] Magli E., "Multiband Lossless Compression of Hyperspectral Images." IEEE Transactions on Geoscience and Remote Sensing, 47(4), 1168-1178, 2009.

[13] Motulsky H. and Christopoulos A., "Fitting Models to Biological Data using Linear and Nonlinear Regression: A Practical Guide to Curve Fitting", Oxford University Press, 2004.

[14] Mamun M. A., Jia X. and Ryan M., "Adaptive Data Compression for Efficient Sequential Transmission and Change Updating of Remote Sensing Images", IEEE Geoscience and Remote Sensing Symposium (IGARSS '09), Cape Town, South Africa, p. 498-501 (IV), July 2009. 